
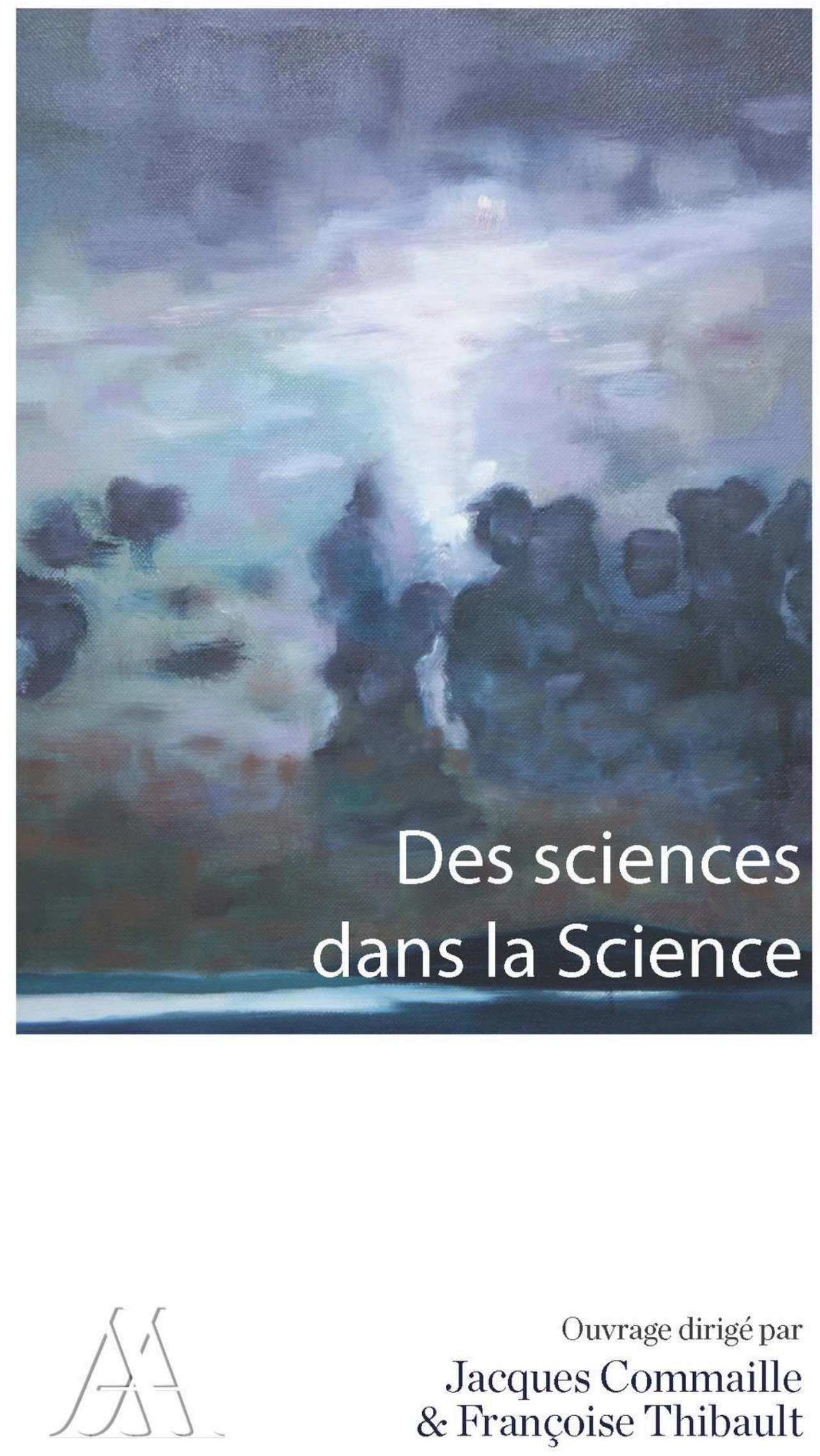


\section{Des sciences dans la Science}

Sciences with the Science

\section{Jacques Commaille et Françoise Thibault}

DOI : 10.4000/books.allianceathena.192

Éditeur : Éditions A. Athéna

Lieu d'édition : Paris

Année d'édition : 2014

Date de mise en ligne : 29 juillet 2015

Collection : Collection ATHENA

EAN électronique : 9791093170107

\section{OpenEdition}

\section{Books}

https://books.openedition.org

\section{Édition imprimée}

EAN (Édition imprimée) : 9791093170008

Nombre de pages : 70

\section{Référence électronique}

COMMAILLE, Jacques; THIBAULT, Françoise. Des sciences dans la Science. Nouvelle édition [en ligne] Paris : Alliance Athena, 2014 (généré le 19 avril 2023). Disponible sur Internet : <http://

books.openedition.org/allianceathena/192>. ISBN : 9791093170107. DOI : https://doi.org/10.4000/ books.allianceathena.192.

\section{Crédits de couverture}

(c) Marianne Van de Logt

(c) Éditions A. Athéna, 2014

Licence OpenEdition Books 


\section{RÉSUMÉS}

Dans un contexte européen où se redéfinissent les grandes orientations de la recherche, une réflexion s'impose sur les principes généraux au fondement des sciences humaines et sociales et sur leur place dans la science et dans la société.

La nécessité d'un dialogue fécond entre les sciences pour faire face aux grands enjeux du monde contemporain, invite à renouer avec la figure de "l'ingénieur " largement empreinte d'une formation ouverte aux humanités.

$\mathrm{Au}$-delà des considérations propres au monde scientifique, les SHS reposent la question du sens même de nos sociétés et de leurs évolutions.

Fruit d'une mobilisation exceptionnelle de chercheurs et enseignants, cet ouvrage remet les SHS à la place qui leur revient et encourage la promotion d'hommes et de femmes de science qui évitent les grands écueils du technicisme, du dogmatisme et du scientisme.

\section{JACQUES COMMAILLE}

Professeur émérite à l'Ecole Normale Supérieure de Cachan et Président du Comité d'orientation de l'Alliance ATHENA

\section{FRANÇOISE THIBAULT}

Directrice de programme à la Fondation Maison des sciences de l'homme et Déléguée générale de l'Alliance ATHENA 

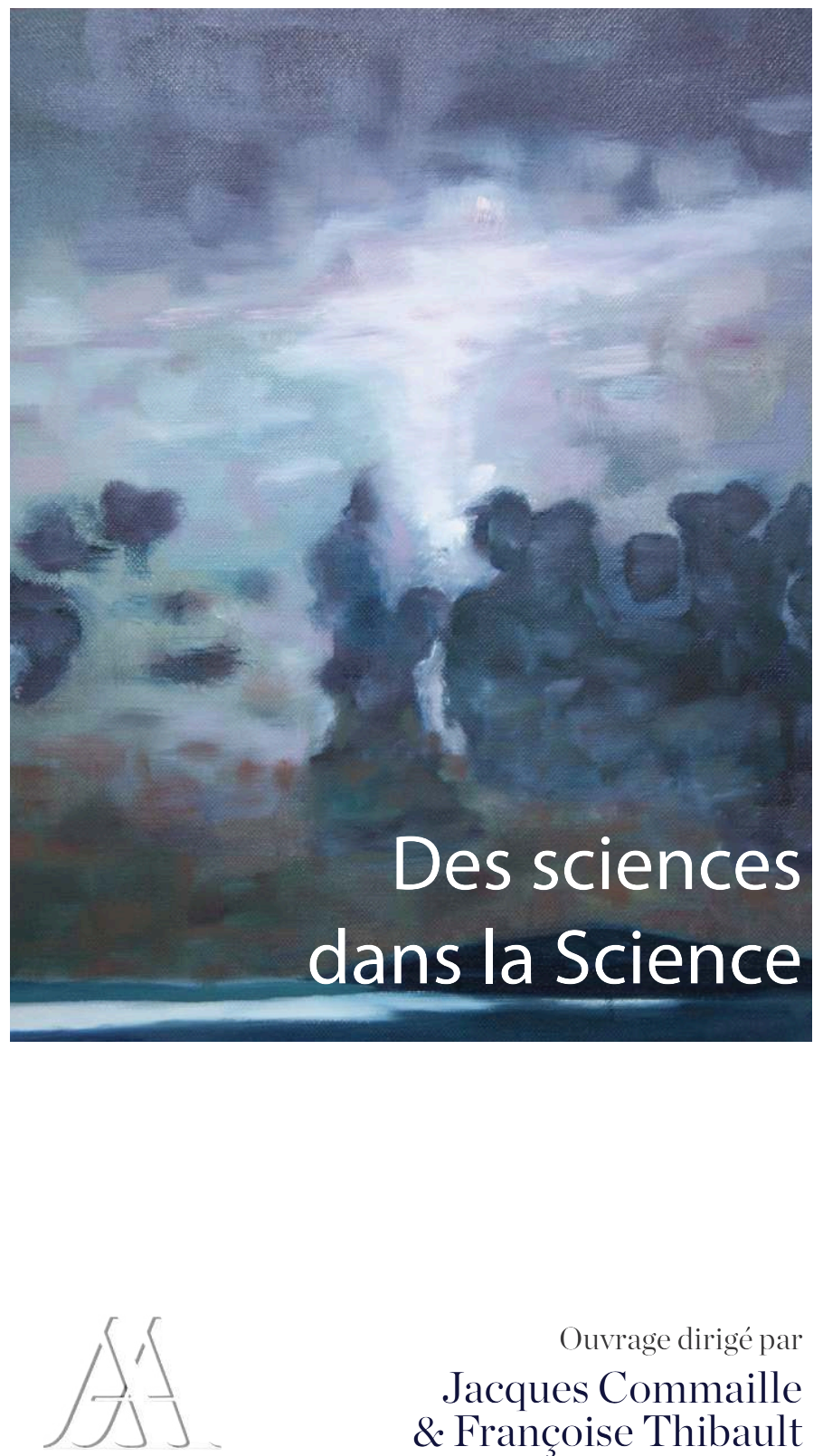

Ouvrage dirigé par Jacques Commaille \& Françoise Thibault 



\title{
Des sciences dans la Science
}

\author{
Ouvrage dirigé par \\ Jacques Commaille \& Françoise Thibault
}

Collection ATHENA 
Collection ATHENA, imaginée et dirigée par Françoise Thibault

Disputatio autour de la politique scientifique pensée comme un art d'organiser et de développer la recherche au bénéfice de la connaissance et des citoyens.

Illustration de couverture : (c) Marianne van de Logt

Responsable de publication : Chloé Lepart

Crédits illustrations : (C) Marianne van de Logt

(C) Alliance Athena, 2014

ISBN 979-10-93170-00-8

ISSN 2425-2379

www.allianceathena.fr

Tous droits réservés pour tous pays 


\title{
Des sciences dans la Science
}

\author{
Ouvrage dirigé par \\ Jacques Commaille \& Françoise Thibault
}

Collection ATHENA 



\section{Préface}

Dans un contexte européen où se définissent les grandes orientations de l'avenir de la recherche, les responsables de l'Alliance ATHENA ont considéré qu'il relevait de leur mission de favoriser une réflexion sur les principes généraux au fondement des sciences humaines et sociales, de leur place dans la science en général en même temps que dans la Cité. Un dispositif a ainsi été mis en place permettant, outre la consultation de représentants des organismes et administrations concernés, l'implication d'une centaine de collègues, sollicités en fonction de leur compétence et de leur discipline, français et étrangers. Si ce dispositif ne saurait prétendre relever d'une logique de démocratie représentative telle qu'elle existe dans les instances de la recherche et de l'enseignement supérieur, il a eu incontestablement comme référence une forme de démocratie participative au service de l'avenir des sciences humaines et sociales. C'est dans ce cadre et dans cet esprit qu'a été élaboré le présent texte, fruit d'une mobilisation exceptionnelle, tant par la qualité des contributions que par celle de leurs auteurs, exclusivement inspirés par la poursuite de l’intérêt général. 
$\mathrm{Si}$, pour l'Alliance ATHENA, ce texte ne saurait prétendre être représentatif des réflexions développées par l'ensemble du potentiel français de sciences humaines et sociales, il constitue à ses yeux une contribution à un débat d'une particulière importance dans une période historique comportant tant d'enjeux pour l'avenir de la recherche. Et ce débat mérite d'être poursuivi, au niveau national et européen, sans qu'aucune instance ne prétende au monopole, dans l'esprit de ce que veut être cette alliance : un espace d'échange, de dialogue au service de l'ensemble des communautés scientifiques concernées.

Dans le texte qui suit, en référence à leurs statuts, les sciences humaines et sociales se donnent à voir dans leur diversité assumée, des humanités aux sciences sociales, considérant que cette diversité n'est pas une hétérogénéité invalidante mais bien une richesse qu'il convient d'exploiter plus encore à l'heure du mouvement de rapprochement de l'ensemble des sciences et pour échapper à la tentation scientiste grandissante et aux diktats du court terme. Les sciences humaines et sociales sont au cœur du processus de formation de l'esprit scientifique. A ce titre, elles sont des sciences critiques, non pas au sens de la simple dénonciation de l'existant mais au sens du dévoilement ou de la perception de ce qui est caché ou minuscule et participe pourtant de la constitution de l'humain et du fonctionnement du monde social.

Les sciences humaines et sociales n'éludent pas la question de leur utilité mais l'intègrent dans une perspective plus large où ne sont pas dissociées cette question et celle des 
conditions de la scientificité grâce à la quête de paradigmes, de théories, de concepts et de méthodes. Les sciences humaines et sociales soulignent avec force que l'exigence de scientificité et du respect des règles de la production de connaissance ne saurait justifier que soit écartée la dimension citoyenne de la recherche, c'est-à-dire la question des conditions de l'information ou même de la participation des citoyens, de leur inscription dans le processus de production, de diffusion et d’appropriation de la connaissance scientifique.

Assurées de leur identité propre, les sciences humaines et sociales réaffirment la place qui leur revient dans les phénomènes étudiées par les autres sciences dans la mesure où tout phénomène étudié par ces autres sciences, toute nouvelle technologie, comportent des dimensions humaines et sociales dont la prise en compte conditionne la réussite des objectifs poursuivis.

Enfin, les SHS entendent apporter une contribution forte à une question majeure qui taraude tant nos sociétés que la science elle-même : celle du sens. Le sens dans ses multiples dimensions, de la compréhension, de la trajectoire dans le temps, de la pertinence, ou de l'éthique.

Telles sont quelques-unes des orientations de la réflexion développée dans le présent texte qui vise précisément à inscrire positivement toutes les sciences humaines et sociales dans la science en général et à leur définir un avenir commun.

Jacques Commaille 



\section{Sommaire}

Les SHS hors de leurs premières frontières

Sociétés, sciences

$\&$ sciences humaines et sociales

Quel régime de connaissance face à la complexité des mondes

Quelle utilité ?

Des sciences avec les sciences.

L'évidence d'une porosité

Conclusion

5 principes d'action pour la recherche en Europe 



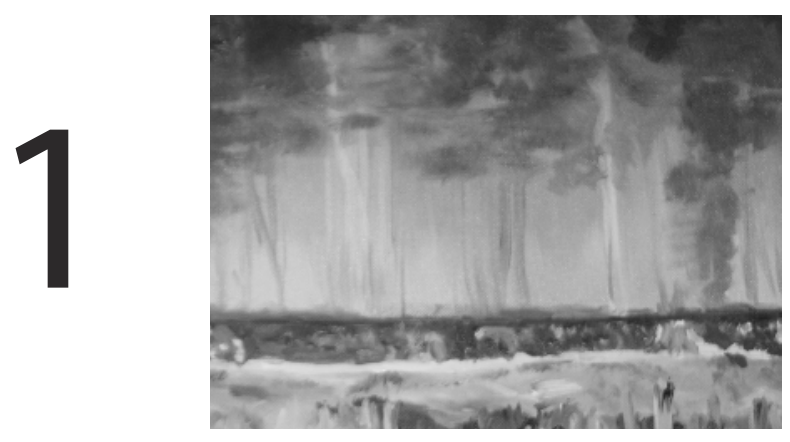

\section{Les SHS hors de leurs premières frontières}

La grande variété des perspectives des sciences humaines et sociales - depuis la philosophie, la littérature, l'étude des religions et des arts jusqu'aux sciences historiques, l'anthropologie, la sociologie, l'économie, les sciences de l'information et de la communication, la science politique - permet à celles-ci d'envisager des convergences, de faire prévaloir ce qu'elles partagent et sont susceptibles de mettre en commun pour contribuer au traitement tout à la fois de questions tenant à la complexité du réel et de l'humain et de questions de nature physique, technologique ou technique, relevant des autres sciences. Cette volonté de mise en commun des connaissances et compétences est fondée sur la conviction que la vieille 
opposition, héritée du XIX ${ }^{\mathrm{e}}$ siècle des sciences de l'esprit et des sciences de la nature, nourrissant le débat entre la compréhension, réservée aux phénomènes humains, et l'explication, propre aux phénomènes naturels, a été depuis longtemps dépassée. Les sciences humaines et sociales, de l'archéologie à la linguistique, sont traversées par des techniques et des questionnements venus des sciences de la nature ou de l'information, tandis que ces dernières sont profondément engagées, de la médecine à l’informatique en passant par la physique, dans des problématiques herméneutiques ou historicistes.

Les sciences humaines et sociales participent pleinement à la redéfinition de ce que sont les sciences au cour de notre société, et elles témoignent de la parenté de leur projet intellectuel avec celui des autres disciplines scientifiques. Il ne s'agit pas ici de promouvoir les SHS mais de consacrer leur inscription dans une certaine idée de la science, comme projet d'élucidation de l'homme, de la société et du monde, sans distinction d'ordre ni de dignité, à partir d'une commune exigence de méthode, de critique et de progrès, au service d'une commune ambition de savoir mais aussi d'utilité sociale.

Les SHS sont des sciences de l'intelligibilité du monde, leurs objets des laboratoires où s'expérimentent des techniques et des méthodes qui peuvent être transposées ailleurs. Il revient donc aux chercheurs en SHS de faire ce travail d'explication. En effet, l'importance des études littéraires, héritées de la philologie, de l’herméneutique ou de la rhétorique, des études historiques et archéologiques ou des sciences sociales ne 
reposent pas seulement sur leur capacité à transmettre une tradition culturelle, à alimenter une ingénierie sociale ou à agrémenter une vision humaniste de nous-mêmes. Elle réside également dans la mise en évidence d'opérations intellectuelles décisives pour des chantiers essentiels de notre monde contemporain. Par exemple, la gestion des flux d'informations, dans sa dimension cognitive mais aussi pratique, depuis l'émergence du big data jusqu'aux problèmes de sélection, d'archivage, de hiérarchisation qui sont posés en aval, est fondamentalement une problématique qui renvoie à des savoirs philologiques, archivistiques et archéologiques. Les sciences cognitives, médicales, informatiques ou physiques ont sans cesse à voir avec des questions d'ordre herméneutique, sociologique ou historique. Les travaux linguistiques ou littéraires les plus complexes, sur les données les plus lacunaires, sont des terrains d'élaboration privilégiés de ces questions, de même que le sont les mondes de l'art, de la philosophie ou de la musique.

Par-delà ces opérations intellectuelles nécessaires, les SHS contribuent à l'intelligibilité du monde par une formation large de l'esprit, par l'appréhension rationnelle et l'élaboration d'une compréhension de la complexité de la réalité, par la construction de sens, celui de l'action humaine et de l'évolution des sociétés. 



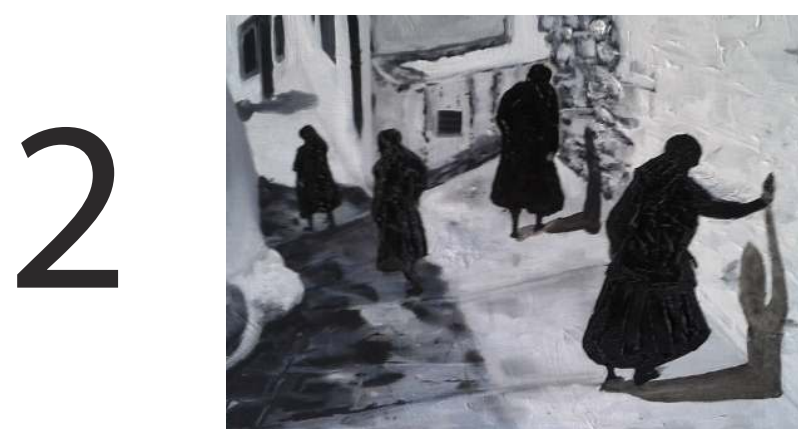

\section{Sociétés, sciences $\&$ sciences humaines et sociales}

Trois évolutions méritent d'être relevées pour qui s'attache à penser la place des sciences humaines et sociales dans leurs relations aux autres sciences et aux sociétés humaines.

La première évolution relève du régime de connaissancepropre des sciences humaines et sociales. D’une part, les SHS ont démontré, ces dernières décennies, leur capacité à travailler de manière interdisciplinaire, notamment pour analyser les questions d'identité collective, de constitution des univers privés en référence aux espaces publics, de structuration et de cohésion sociale et politique, de processus de construction de nouvelles citoyennetés. D’autre part, les SHS ont apporté de 
multiples preuves de l'indissociabilité des enjeux « naturels » (ressources, climat, etc.) ou technologiques, et des enjeux concernant la subjectivité et l'agentivité des individus, prenant en compte leur modes de constitution du collectif, avec leurs visions du monde inspirées du passé, immergées dans le présent et marquées par des rapports différents à l'avenir. Les efforts accomplis pour affirmer l'identité et défendre les frontières des territoires SHS ont fait place à des perspectives de travail collectif où prévalent les objectifs de connaissance à atteindre en référence aux enjeux auxquels nos sociétés actuelles sont confrontées. C'est là, par exemple, tout le projet des Maisons des Sciences de l'Homme initié en France au début des années 1960 et généralisé à la fin des années 1990.

La deuxième évolution renvoie à la question aigüe du statut de la science. L'affrontement est vif entre une science dite « de la nature » qui tente d'intégrer les dynamiques humaines et une science de la mesure, plus forte que jamais, qui réduit l'humain à quelques phénomènes observables et quantifiables. Pour les premiers, l'humain fait explicitement partie de la chaine causale de la nature, par les transformations qu'il suscite, volontairement ou involontairement, dans le monde qu'il habite. La science est alors considérée comme une production humaine, et une activité qui doit être en permanence discutée au nom de sa responsabilité à l'égard des humains susceptibles d'être affectés par elle. Cette science tend à donner aux SHS une place majeure renouant en cela avec les fondements anciens. Pour les seconds, l'humain est un objet comme un autre, qu'une science toute puissante serait en mesure, à terme, 
de déchiffrer totalement. L'immanence d'une telle science la met à l'abri d'un questionnement permanent et de nombreuses disciplines des SHS se voient refuser le statut de science au nom d'un trop grand voisinage avec le subjectif et le créatif.

La troisième évolution concerne l'intégration des progrès scientifiques et technologiques dans un nouveau modèle de régulation des sociétés, ce dernier étant considéré dans ses dimensions sociétales, économiques, juridiques et politiques. L'avancement des sciences, ses effets multiples sur l'humain et sur les sociétés, ne suffisent pas à définir l'idée de progrès, encore moins à susciter la confiance en l'avenir. Un progrès qui n'intègre pas l'ensemble des composantes d'une société, ni ne se confronte au monde dans sa globalité, ne peut plus être considéré comme tel. Il suscite, plus que par le passé, méfiance, hostilité, disqualification voire rejet (pouvant s'accompagner du rejet du modèle de société démocratique), si ne sont pas posées les questions concernant le sens des problèmes rencontrés et définies les options possibles pour les résoudre.

C'est en ce sens que s'affrontent, dans les sociétés humaines, des conceptions radicalement divergentes de la modernité et de la place qui doit être accordée à la science. Al'image d'une science omnipotente peut répondre un refus de science au bénéfice des croyances. Pourtant, le processus historique de supranationalisation et de transnationalisation en cours, les déséquilibres dans l'occupation des espaces (urbanisation croissante, fragmentation des espaces sociaux, etc.), dans les processus du vivre ensemble (avènement des sociétés « singularistes », 
incertitudes des instances de socialisation, etc.), dans le partage des ressources et les problèmes écologiques, actuels et prévisibles, rendent indispensable l'exercice d'une science globale ouverte et réflexive qui transcende les découpages disciplinaires hérités des siècles passés et confère aux grands acquis des sciences humaines et sociales un statut de savoirs communs à tous les chercheurs. 


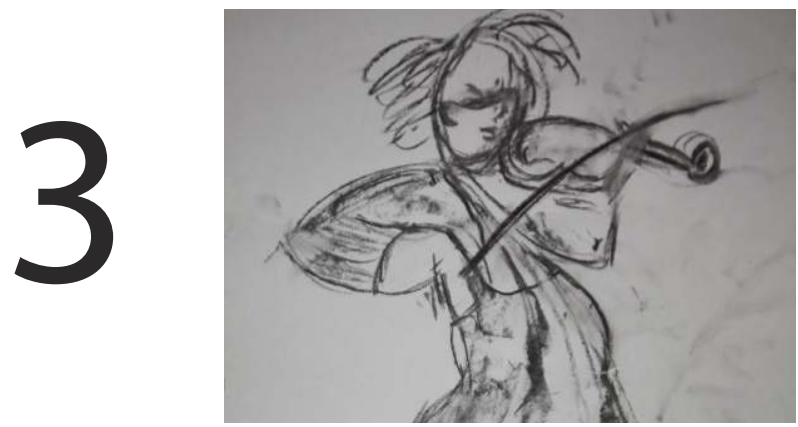

\section{Quel régime de connaissance face à la complexité des mondes?}

Le contexte historique d'un monde en mutation justifie plus que jamais l'importance des SHS et du rôle qu'il leur revient de tenir. En effet, les bouleversements du monde ont rendu ce dernier à la fois énigmatique et opaque : énigmatique parce que la plupart des grands repères politiques de l'aprèsguerre, les systèmes techniques, la mondialisation des grandes entreprises, l'évolution des peuples, leur migration et leur mobilité croissante, le développement d'entités supranationales, les régimes du savoir et de l’innovation ont muté; opaque parce que ces transformations ne s'étudient pas avec les instruments scientifiques existants et exigent des configurations de 
recherche nouvelles dans lesquelles les sciences humaines et sociales se sont d’ores et déjà engagées.

Dans ce contexte, évoquer un nouveau régime de connaissance des sciences humaines et sociales, c'est souligner une triple exigence préalable à toute inscription de ce domaine scientifique dans une programmation à moyen et à court terme en fonction de « défis » auxquels nos sociétés sont confrontées. Cette triple exigence préalable consiste à revenir sur : (i) l'identité propre des SHS, (ii) l'exigence de réflexivité qui touche leur socle épistémologique et leurs développements théoriques, (iii) l'implication des SHS dans la mobilisation des connaissances concernant d'autres sciences, et ce, en amont des agendas technologiques et scientifiques.

\section{Une identité plurielle}

Les SHS présentent une riche variété d'hypothèses, de paradigmes, de méthodes et de pratiques pluralistes qui est leur force. Cette diversité s'observe dans les objets, les théories et les méthodes investies par les différentes disciplines, comme dans la résonance publique qu'elles trouvent constamment hors des cercles académiques. Ce pluralisme est un atout considérable pour une société qui se veut elle-même pluraliste et novatrice. Cet atout est d'autant plus important s'il est assumé et valorisé comme tel, comme une ressource pour les démocraties post-industrielles, pour les aider à se penser et à se situer. 
Les sciences humaines et sociales sont marquées depuis leur naissance par la transformation des significations vécues en un univers de significations objectives. Cette caractéristique rend intéressant le fait que l'on parle de sciences humaines ET sociales. La tentative est bien de rendre compte, le plus exactement possible, de la réalité de l'action humaine dans le monde tout en laissant ouverte la question de l'être. La philosophie a joué et continue de jouer un rôle pionnier et essentiel grâce à la rigueur des questions qu'elle pose, à la démarche scientifique et aux ouvertures interdisciplinaires qu'elle offre au sein des SHS et, bien au-delà, apparaissant comme un interlocuteur «naturel » des sciences de la vie ou des sciences de la matière.

L'autonomisation des sciences humaines les unes vis-à-vis des autres (philosophie, sociologie, histoire, linguistique, etc.) correspond à une logique de spécialisation dans la diversification du travail intellectuel, au développement des techniques d'enquêtes empiriques, comme des méthodes de traitement des données.

Les mérites de cette diversité se traduisent de plusieurs façons et il n'en sera donné que quelques illustrations :

- les recherches sur les pratiques et activités artistiques, linguistiques, culturelles sont attachées à la compréhension des évolutions/transformations de nos sociétés et de nos cultures dans l'ensemble du monde, depuis l'étude des primates non-humains et de la diversité culturelle au 
cours des temps, afin d'appréhender l'émergence de nos anatomies, de nos comportements (locomoteurs comme la bipédie, alimentaires, cognitifs associés au langage, etc.), de nos structures, de nos savoirs ;

- les sciences historiques sont à même d'apporter une profondeur chronologique, ample ou resserrée, aux phénomènes contemporains. Elles rappellent que les sociétés humaines sont déjà passées par des phases majeures de transformations, largement mues par des innovations techniques et scientifiques. Les méthodologies qu'elles mettent en ouvre cherchent à objectiver le passé afin d'en restituer la complexité. Elles offrent à la société les moyens de sorienter parmi les interprétations possibles de ce passé commun. Les sciences sociales, de façon générale, se consacrent aux nouvelles réalités et aux nouvelles représentations des territoires, aux nouvelles formes d'appropriations des espaces (par exemple, l'urbanisation du monde), aux formes différentes de vivre ensemble associées aux menaces pesant sur la cohésion sociale, aux figures inédites de la gouvernance et de la mobilisation politique et aux interrogations sur les procédures canoniques de l'établissement de la démocratie, aux ressorts d'une vie économique se donnant à voir sous la double face de Janus : celle de la surpuissance, celle des déséquilibres sociaux et politiques qu'elle provoque...

Loin d'affaiblir les SHS, cette variété des approches et des méthodes est devenue un atout majeur dès lors que, tout en 
spécifiant la singularité de leurs socles épistémologiques respectifs, elles en sont venues depuis quelque temps à se confronter entre elles et avec les autres sciences. Leur pluridisciplinarité dans le cadre de ce travail de mobilisation inédit conduit à des configurations novatrices et à des réponses concrètes aux interrogations des sociétés actuelles, avec une attention indispensable aux exigences nouvelles du débat démocratique dans lequel le citoyen est porteur des enjeux («stakeholder ») de la science.

\section{L'exigence de réflexivité}

Ce travail d'identification s'accompagne d'un travail réflexif permanent sur les conditions de production et de mise à l'épreuve des savoirs eux-mêmes et sur leur statut dans une société démocratique. La nouvelle configuration des SHS doit être marquée par cette aptitude à se livrer à ce travail réflexif. Une place doit être ainsi laissée à ces réorientations et aux redéfinitions parfois radicales susceptibles d'en résulter pour les régimes de connaissance, ceci afin que puissent être appréhendés des phénomènes et des systèmes complexes, affrontés des « défis », identifiés et surmontés des problèmes, grâce à l'affinement d'outils critiques - au sens savant permettant d'échapper à l'illusion du devenir d'un monde enchanté parce qu’on le nomme comme tel ou de sombrer dans la facilité des dénonciations idéologiques. 
Une des contributions des SHS est d'avoir montré que le phénomène économique ne peut être confondu avec les modèles de pensée de la discipline éponyme. Il faut donc clairement distinguer le phénomène économique des modèles que l'on appelle « économiques ». Cette opération va bien plus loin que la distinction traditionnelle entre science économique et science sociale. Cette division tend à conserver la stricte correspondance entre phénomène et modèle, alors qu’il faut accepter qu'il y ait des modèles sociaux du phénomène économique comme il y a des modèles économiques de phénomènes sociaux. Par exemple, le phénomène de la « titrisation » financière, un des mécanismes amplificateurs de la débâcle bancaire des subprimes, est incompréhensible si l'on se contente de se demander si ces «titres » ont été «bien ou mal évalués » par un modèle économique. Il importe autant de comprendre comment de multiples intervenants et porteurs d'enjeux, dont la liste est elle-même un objet de recherche, ont pu générer des règles de gestion, à l'échelle de la planète, qui ont rendu la «titrisation » possible malgré ses effets catastrophiques. Ce n'est que lorsque l'ensemble de cette analyse est conduite que la question du «bien ou mal » évalué peut être posée et relativisée selon les différents protagonistes du phénomène.

La mutation épistémologique qui s'opère est donc du même ordre que la naissance de la chimie pour l'étude de la «matière». Cela a conduit à bien distinguer le phénomène " matière » des modèles analytiques du physicien. Cette mutation explique aussi avec le recul, pourquoi les multiples tentatives 
d'élargissement de la science économique par adjonction d'autres disciplines n'ont pas modifié les hypothèses centrales de cette discipline. Il est plus scientifique de reconnaître que le phénomène économique relève de plusieurs modélisations venant de l'économie, de la sociologie, des sciences de gestion ou d'autres disciplines comme la philosophie. Cette mutation s'illustre particulièrement dans deux domaines qui sont des défis majeurs des sociétés : la crise de l'entreprise et les mécanismes de l’innovation.

\section{L'implication}

L'innovation intellectuelle, l'intelligibilité que nos sociétés ont d'elles-mêmes, diachroniquement et synchroniquement, la construction et le développement de communautés démocratiques, ouvertes et capables d'intégrer leur passé dans leur présent sans en subir la contrainte sont le résultat décisif de la recherche en SHS - et c'est cette utilité sociale qui a fondé la place des sciences humaines et sociales, au XVIII et au XIX ${ }^{\mathrm{e}}$ siècle, dans l'élan né des Lumières et poursuivi lors de la naissance des disciplines modernes.

C'est en ce sens qu'en SHS, la recherche supposée « appliquée » est bien davantage une recherche «impliquée ». Les catégories et les hypothèses de ces sciences doivent être mises à l'épreuve sur les situations historiques, sociales, sur les interactions entre humains. Ces derniers se réapproprient alors ces catégories et hypothèses qui deviennent éléments de la vie 
sociale. Ce sont ces capacités d'implication, de prise des hypothèses sur la vie sociale, qui assurent in fine la validité des recherches. Même dans les théories les plus formalisées (ainsi en économie), le banc-test des fondements théoriques reste de ce type. La recherche « fondamentale» ne peut donc prétendre à une simple « application » qui serait ancillaire, et les recherches supposées «appliquées » à tel ou tel problème de société n'ont de fécondité que comme recherches « impliquées » qui permettent aussi de réviser les fondements des problématiques.

Pour ne prendre qu'un exemple, l'innovation exige : a) que l'on réfléchisse à cette mise à l'épreuve que pourra mettre en évidence la recherche impliquée, b) sans que la créativité des processus d'innovation ne soit bloquée d'avance par des préjugés sur les contraintes sociales, puisque c) l'innovation vient de la société autant que de la recherche, qui doit donc être attentive aux modifications de contraintes que peuvent apporter ces germes d'innovation sociale. La créativité suppose donc ce que certains ont appelé une « recherche libre ». En effet si les hypothèses de recherche se calquent sur les représentations présentes des problèmes sociaux, leur conformité aux situations sociales ne leur apportera aucune validation, puisqu'elle sera en fait présupposée au lieu de permettre une mise à l'épreuve. 


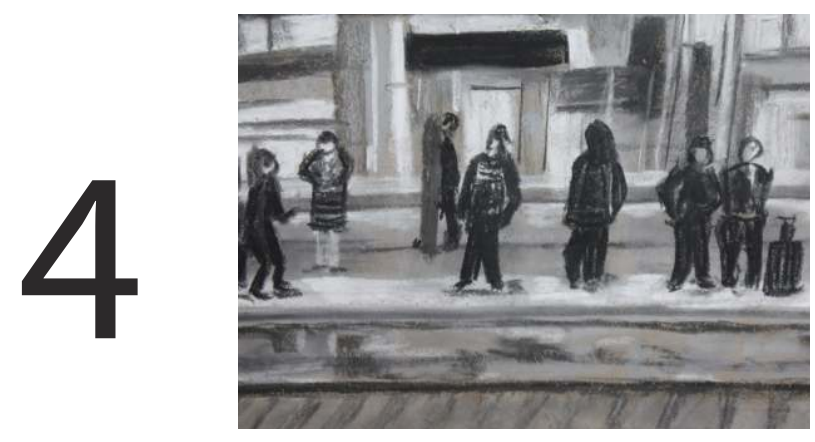

\section{Quelle utilité ?}

\section{Les figures de l'utilité}

Beaucoup a été écrit ou dit sur la société et l'économie de la connaissance. On a pu résumer cela par une triple hélice représentant le transfert de connaissances du laboratoire à l'usine ou au bureau jusque dans la vie quotidienne. Dans ce mouvement transformateur, les producteurs de connaissance sont soumis à de fortes perturbations et leur rôle est tout aussi essentiel que changeant, se déplaçant sans cesse. La triple hélice introduit une rupture dans la manière d'envisager ce que sont les SHS et leur rôle alors que l'on s'est un peu 
trop vite arrêté à l'idée de transfert comme l'application de la conception vers la pratique.

À cet égard, les postures d'intervention du savant en sciences humaines et sociales sont devenues composites : qu'il s'agisse de l'« expert », du «traducteur », qu'il s'agisse du « chercheuraction » ou du «chercheur engagé ». Les facettes d'intervention du chercheur en SHS, la diffusion de ses hypothèses et ses conclusions sont multiples. Nul besoin par conséquent de s’inquiéter de l'applicabilité des apports des SHS et de leur mobilisation dans la relève des défis.

\section{L'exigence d'une stratégie du détour}

Toute prise en considération de ces niveaux dans le processus qui va de la production de connaissance à ses usages conduit finalement à considérer que, pas plus que les autres sciences, les SHS ne peuvent être guidées par un principe d'utilité, a fortiori quand ces utilités sont définies à courte vue, en fonction des intérêts de la seule période en cours.

Au même titre que les autres sciences, l'utilité de la recherche peut découler d’une «stratégie du détour ». Cette «stratégie du détour », bien documentée dans le développement cognitif, passe ici par la recherche fondamentale, la construction de théories, l'emprunt des voies incertaines et de configurations analytiques nouvelles, les rencontres improbables avec d'autres disciplines avant de revenir éventuellement sur l'action. 
Au même titre que pour les autres sciences, les catégories de la connaissance doivent pouvoir se construire de façon autonome par rapport aux catégories d'une pratique, d'une action bornée par les représentations en cours, fut-elle publique, européenne ou internationale, inspirée par la promotion ou la défense d'une cause.

\section{L'invention de nouveaux modèles de sociétés}

La valeur des SHS se situe dans leur vertu explicative, analytique, compréhensive, créative, projective. Elles sont intrinsèquement des sciences impliquées et non appliquées. Assumer cette valeur, comme nous l'avons vu supra, revient à s'autoriser l'invention de nouveaux modèles de société, de vivre ensemble et de créations.

La situation des disciplines concernées leur impose, selon les cas, de critiquer ou d'accompagner la commande publique. Dans les situations les plus favorables, les sciences historiques répondent à la demande en accompagnant et en critiquant la réalisation des projets orientés par un pouvoir public (national, régional ou européen). Pourtant, dans une démocratie, les décideurs politiques gagneraient à entendre les propositions, les hypothèses fondées, les explications appuyées par des méthodologies (que les scientifiques opposent à une conception étroite et instrumentale de la science), et ce, dans une relation qui devrait être de confiance mutuelle. 
De façon générale, au-delà des sciences historiques, les savants ont toujours trouvé une légitimité auprès des politiques. Cette place ne relevait pas du principe d'utilité (bien que l'on ait pu parler de «technologies politiques ») mais de la reconnaissance de leurs régimes de connaissance spécifiques qui fondaient leur pluralité (et donc le pluralisme complémentaire de savoirs), et de la variété de leurs méthodes et approches, qui assuraient leur capacité de diagnostic relevant d'une expérience de la critique (au sens de crisis : décider), de leur capacité d'analyse et de définition assurant les conditions du débat démocratique et la diversité des points de vue.

\section{L'interaction société, recherche et formation}

Dans les relations entre recherche et action, ce qui vaut pour le rapport au pouvoir politique ou à la commande publique vaut pour le rapport aux postures engagées provenant de la société : les rapports de genre, de la diversité des orientations sexuelles, des situations coloniales et postcoloniales, de l'alarme environnementale ou encore de la demande d'une ouverture des sciences économiques. Ces questions ont inspiré des champs de recherche intégrés au système de l'enseignement supérieur et de la recherche au prix de mobilisations de type militant. Les chercheurs ont forcé les portes de l'université et légitimé ces nouveaux champs de recherche, qui demeurent toutefois souvent vulnérables. On est là confronté, sous réserve de l'exercice de vigilance critique, à un mode de régulation normal du système d'enseignement supérieur. Tout 
système de recherche et d'enseignement fermé à de telles pratiques est un système en voie de dissolution. La difficulté pour l'Université est alors de ne pas produire de nouveaux spécialistes œuvrant dans des cercles relativement fermés mais d'être en capacité de faire circuler ces savoirs au sein de nombreux enseignements.

Plus encore aujourd'hui, une formation aux sciences humaines et sociales est indispensable à l'ensemble des étudiants pour maîtriser la complexité des mondes actuels, opérer les mises à distance des objets étudiés et permettre le dialogue inter sciences. Les qualités d'abstraction, si puissantes soientelles, sont insuffisantes pour former l'esprit scientifique d'aujourd'hui. Il y a une "somnolence du savoir" sans remise en question permanente du regard sur l'objet étudié et un esprit bien formé à l'abstraction n'est pas à l'abri des vestiges de croyances anciennes. De grands mathématiciens ont fait la preuve, depuis fort longtemps, que l'excès de "mathématisme" conduit à des mesures sans pertinence et à des descriptions quantitatives insensées. Il est ainsi impératif de mettre un frein au processus de spécialisation à l'œuvre dans les cursus universitaires qui s'accompagne de la disparition des humanités.

\section{La distance critique et l'impératif temporel}

C'est aussi, pour les SHS, à partir d'une conception exigeante et critique de la recherche, lorsqu'elle se projette ou lorsqu'elle 
est projetée dans l'avenir, que l'on échappe au syndrome du wishful thinking, particulièrement pervers quand on part de problèmes que les autres disciplines ne savent pas résoudre. La prédominance des cadres de la recherche sur projet laisse implicitement supposer que des SHS efficaces doivent être en mesure de trouver les solutions aux problèmes qui ont une dimension humaine et sociale et supprimer les difficultés en quatre ans. Le recours à des recettes déjà disponibles est encouragé alors que le besoin est au contraire de questionner continûment les préalables : ceux relatifs aux faits, aux interrogations, aux problèmes qui sont donnés à voir.

Il est souvent plus pertinent de rechercher les sources de déséquilibre plus que les équilibres, les sources de conflits que de concorde, d'emballements de violences plutôt que d'apaisements, les sources d'émotions irrationnelles plutôt que raisonnées, ou les obstacles à des coopérations sociales plus que leurs recettes de réussite. La non analyse des résistances, des échecs, des blocages, ne peut que favoriser le discours incantatoire en faveur d'un monde en progrès et n'aboutit finalement qu'à un constat d'impuissance. En un mot, il est indispensable de disposer du temps nécessaire à l'analyse des dynamiques que représentent les contradictions et les tensions inhérentes à la constitution et au fonctionnement des sociétés.

Les thématiques urgentes n'ont de chance d'être relevées que si les fondements culturels constitutifs de l'identité des sociétés, de leurs résistances, de leurs dynamiques historiques, sont 
analysés, si les problèmes économiques et sociaux associés à ces défis sont disséqués et trouvent ne serait-ce qu'une solution partielle. Il importe donc de préciser la nature de ces problèmes, parmi lesquels la déstructuration des relations sociales et des pouvoirs induite sous l'effet conjoint de la mondialisation des échanges et des transformations scientifiques et techniques.

La langue doit faire l'objet d'une vigilance particulière. Pour ne prendre que quelques exemples en matière d'environnement, parler de « changement climatique» au lieu de « réchauffement climatique », d'« adaptation au changement climatique » au lieu de « lutte contre le réchauffement» ou encore se préoccuper de « la prise de conscience environnementale des acteurs économiques et des citoyens »sans se pencher sur les refus ou l'impuissance à passer à l'acte des politiques ou sur les pressions de certains secteurs industriels, porte le risque d'euphémiser l'ampleur des résistances à l'œuvre.

La recherche doit prendre le temps de cette distance langagière, le chercheur n'est pas l'expert. Aux questions attendues, il préfère les voies nouvelles. Penser par exemple les relations entre la protection de l'environnement et le développement économique, c'est laisser entendre a priori qu’il y a deux domaines séparés, alors que la question pourrait être posée de savoir si, précisément, l'économie ne devrait pas être encastrée dans l'écologie et dans les formes de vie humaines et non-humaines, au sens où tout enjeu de relation avec notre cosmos doit faire l'objet d'un calcul, mais d'un calcul avec sa 
dimension politique, c'est-à-dire débattu, où les valeurs et les visées ne sont pas séparées des analyses scientifiques.

La logique de l'équilibre risque ici de reproduire le stéréotype de la protection de l'environnement contre le développement économique alors que c'est leur coopération qui devrait être mise en évidence puisque c'est le terrain d'une meilleure gestion des ressources et d'une innovation guidée par le principe même de préservation de l'environnement.

Autre exemple dans le domaine de la santé : il convient de se donner les moyens d'interroger bien des présupposés des recherches en cours (comme d'ailleurs pour tout autre domaine de recherche), leurs modes d'interaction avec les patients, les modes de vie de ces derniers et les systèmes de soin de façon à explorer de nouvelles pistes. De la même façon, bien des recherches cliniques qui ne prendraient pas en compte et ne testeraient pas la place du patient dans cette boucle seraient en quelque sorte incomplètes car elles feraient le choix d'un schéma thérapeutique a priori qui a pourtant montré ses limites. De nombreux sujets, comme le traitement du SIDA, ont montré l'importance de la participation des publics pour le progrès de la connaissance.

Ces exemples appellent donc les SHS à inscrire, dans la durée, une double posture. Par la première, elles sont, comme toute science, productrices de données et d'analyses sur tous les phénomènes qui ressortent du social. Par la seconde, elles mettent en lumière les différents régimes de connaissance 
existant dans une société. Ni approbation, ni contestation pure, elles travaillent au déchiffrement des interprétations, des controverses, des pratiques afin de conforter la délibération publique. L'adoption d'une telle distance critique vise à une cumulativité du savoir susceptible d'ouvrir de nouveaux champs de recherche, au plus près des transformations sociales, économiques, politiques, et de venir innerver la sphère publique.

\section{Nourrir l'idéal démocratique}

De façon plus générale, le statut des transformations scientifiques et technologiques par rapport aux citoyens mérite d'être analysé autrement que dans une perspective top down. C'est ainsi que l'approche par l'acceptabilité, et non par l'appropriation et la participation des citoyens, se révèle non seulement misérable et méprisante, mais inefficace. Bien des problèmes majeurs actuels relèvent d'une déconnexion entre les centres de décision et de régulation et les milieux de vie, et d'une implication insuffisante des citoyens. Poser le problème de la participation en termes du « citoyen à éduquer » est un parti-pris politique et normatif majeur et un obstacle à la compréhension de ce qui est en train de se dérouler.

L'élévation du niveau d'éducation, l'apprentissage de la contribution via les réseaux numériques, la diffusion massive et la disponibilité d'expertise contradictoires constituent autant de signes d'un changement majeur pour la prise de décision. 
L'incertitude scientifique et technique n'est pas un obstacle mais le ressort même de l'activité scientifique et l'impuissance politique notoire devant les crises contemporaines renforce ce sentiment d'incertitude. Les analyses purement rationalistes des processus qui conduisent de la science à la décision et l'action font face aux limites de la connaissance (known unknowns / unknown unknowns), aux constructions de l'ignorance, aux difficultés de la prise de décision en contexte d'incertitude et de controverses. Les décisions publiques sont confrontées à de nouveaux critères : bien commun, utilité sociale et environnementale, responsabilité. Elles doivent intégrer et reconnaître la compétence des citoyens : la démocratie se définissant comme gouvernement de la participation égale de tous, sans distinction relative à leur détention de connaissances dans les domaines qui font l'objet des décisions collectivement prises. Elles doivent prendre en compte le développement, depuis des décennies, de dispositifs de consultation multiples et concurrents, s'efforçant d'inclure, dans l'élaboration de critères pertinents et rénovés, des responsables publics, des experts scientifiques et des citoyens ordinaires.

L'aide à la décision dans la construction et l'évaluation des politiques publiques ne peut donc plus avoir recours à une expertise purement technique ni à des outils d'analyse qui ont montré leur inadéquation aux contextes dont les impératifs épistémiques, éthiques et politiques deviennent forts. 
Il devient essentiel de prendre en compte cette situation pour imaginer d'autres formes de participation qui prennent au sérieux les capacités contributives des publics qui ne souffrent ni d'irrationalité ni d'informations insuffisantes mais de pertes de confiance dans des autorités perçues comme au mieux impuissantes, au pire complices (par exemple de malversations financières et écologiques). L'étude des méthodes expérimentées pour récupérer du pouvoir (empowerment), c'est à dire de la capacité d'agir, par les collectifs divers, pour composer des solutions nouvelles pour des problèmes qui sont objets de controverses, doit être renforcée dans un dialogue avec les scientifiques, les ingénieurs, les décideurs et à condition de donner au citoyen le temps d'expérimenter et d'apprendre.

Le développement actuel de travaux pluridisciplinaires de sciences sociales, notamment dans la recherche juridique, nous offre en la matière un exemple parmi d'autres. Ces travaux portent sur les formes de mobilisations de la ressource du droit - civil, pénal, commercial, de la propriété industrielle, international, etc. - ou de celle représentée par la justice (juridictions nationales et supranationales) sur des questions traitant de la maîtrise de certaines évolutions scientifiques (par exemple, en physique, en biologie, en génétique, dans le domaine agro-alimentaire, dans celui de l'environnement, etc.). Ils rendent compte d'aspirations croissantes exprimées par des formes organisées de la société civile, au niveau national comme au niveau supranational, à être associées à ce que fait la science et aux effets de ce qu'elle produit. 
La recherche française porte actuellement de l'attention à des courants théoriques comme celui du law consciousness (en référence à un droit constitutif de la société, inscrit dans la vie quotidienne des citoyens) ou du cause lawyering (la prise en charge par les professionnels du droit de « causes » soutenues par des mouvements collectifs éventuellement issus de la société civile mondiale) ou encore à des demandes, en matière de santé, de protection juridique de savoirs ou de produits issus des sociétés traditionnelles face à des entreprises pharmaceutiques.

Le développement des recherches sur le genre et les sexualités, très important depuis quelques années en France, est également poussé par l'urgence démocratique de la prise en compte des femmes, comme « donnée » dans la constitution des connaissances (en histoire, en droit...), et comme élément d'égalité. Le genre est un cas, exceptionnel à trois titres, de concept scientifique constitué par l'interdisciplinarité même et par les croisements disciplinaires, créé par la transformation d'un mouvement social en domaine de recherche et d'analyse critique d'une situation d'inégalité globale, et capable de s'étendre des SHS aux autres sciences : l'intégration de la donnée « différence de sexe » dans le domaine de la santé (tests sur les médicaments), mais aussi de l’ingénierie et de l'environnement a été source de plusieurs importantes avancées récentes. Les controverses autour de la dite « théorie du genre » ont permis d'illustrer certaines tendances du scientisme, prompt à dénoncer comme «idéologie » voire 
« antiscience » des innovations scientifiques qui ont un effet de critique sociale et de démocratisation.

Ces nouvelles orientations de la recherche sont illustratives du travail d'analyse qu'appellent les nouvelles exigences démocratiques face aux changements scientifiques ou techniques. 



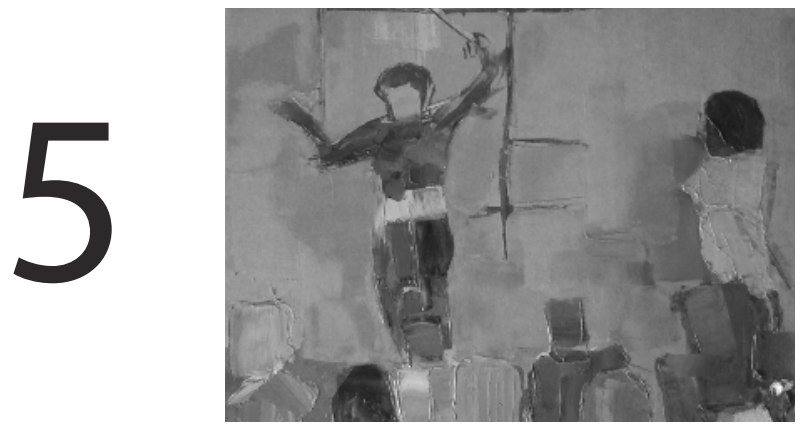

\section{Des sciences avec les sciences L'évidence d'une porosité}

L'implication potentielle des SHS dans la production de connaissances relève d'une telle ampleur qu'elles se situent non pas à côté des autres sciences mais en lien étroit avec elles.

Les communautés SHS, grâce à leur capacité de prise en compte de l'incertitude et de la complexité, ont à prendre place dans une politique scientifique plus large que leurs domaines propres parce que les objets sont frontières et hydrides. La prudence (au sens aristotélicien) est nécessaire à la politique de l'innovation. En effet, les SHS ne sont pas un domaine périphérique, elles n’interviennent pas après coup pour donner un «supplément d'âme » aux autres sciences 
ou être assignées à la fonction de simple outil d'acceptabilité de ce que le progrès scientifique ou technologique impose. A partir de leur spécificité, elles peuvent participer des avancées des autres sciences. Beaucoup des grands programmes ne sont réalisables qu'avec les SHS (notamment dans le domaine de la santé, de l'énergie, du climat...).

Les sciences dont les SHS, dans le long mouvement d'idées du dernier siècle, ont successivement développé des spécialisations permettant un développement de connaissances approfondies puis des approches où la complexité, l'interaction, la non linéarité et la prise en compte simultanée du temps et de l'espace ont nourri la pensée et la construction de connaissance.

Les SHS disposent d'outils formels pour penser des complexités. Elles apparaissent ainsi comme les mieux placées pour fournir des échelles de comparaison fondées sur des méthodes rigoureuses et distinctes en fonction des objets et des disciplines. Elles portent en elles, de plus en plus, la capacité de signaler et de distinguer les possibilités d'interprétations divergentes des résultats obtenus que les théorisations ont tendance à négliger.

Les sciences sont interpellées le plus souvent suivant des dimensions qui sont de la compétence des SHS. Pourtant, on constate que les approches technologiques, ou relevant des sciences expérimentales, l'emportent régulièrement, bien que les principaux problèmes d'ordre naturel, physique, 
technologique auxquels sont confrontées nos sociétés, ces « défis de société », comportent toujours des dimensions sociales, économiques ou politiques dont la non prise en compte peut conduire à l'échec ou à l'impuissance.

Pour ne prendre que trois exemples, les questions d'environnement sont abordées majoritairement sous l'angle des problèmes physiques ou biologiques. Or, leurs principaux déterminants relèvent de l'activité humaine et les enjeux que ces questions comportent ne peuvent être dissociés des questions de gouvernance (y compris les conditions d'acceptabilité des politiques d'action publique), et doivent tenir compte des diverses échelles, du global au local.

De même, l'exploitation des terres et des ressources naturelles en vue de l'alimentation ou pour produire de la biomasse nécessite des arbitrages. Ces questions sont politiques et demandent des choix qui seuls peuvent garantir au minimum que toutes les options restent ouvertes.

Cette mobilisation multiple des SHS sur les différents enjeux est d'autant plus nécessaire qu'elle doit se confronter à la complexité et à l'interdépendance de ces enjeux. Par exemple, les questions qui ont trait à la gestion des ressources dans une perspective de développement durable ne peuvent être abordées et traitées qu'en prenant en compte les interactions et les «boucles de rétroaction » qui se nouent au sein de systèmes complexes (écosystèmes et systèmes socio-économiques) et entre ces systèmes. Outre une approche interdisciplinaire, 
à l'interface avec les sciences de la vie et les sciences de la terre, ces questions exigent de promouvoir une authentique transversalité des problématiques.

Le défi de la sécurité alimentaire fournit un autre bon exemple. Il ne peut être relevé sans intégrer la préoccupation d'une gestion durable des ressources, en terre et en eau, des espaces agricoles de la planète. Or, le développement rapide et incontrôlé d'une agriculture financiarisée (ou de firme), sous des formes diverses (land grabbing, spéculation sur les récoltes et les produits, intensification à outrance, etc.), est un vecteur de dégradation des ressources (terre, biodiversité, produits agro-alimentaires) ainsi que du bien-être et de la santé des populations. C'est toute la chaîne des interactions entre écosystèmes et systèmes économiques qui doit ainsi faire l'objet d'investigations.

Le défi de la transition énergétique nécessite de développer un agenda SHS qui prenne acte de la dimension indissociablement socio-technique des technologies afin de centrer l'analyse sur les différentes dimensions des processus de formation des potentiels technologiques. Il s'agit de porter un regard large sur les options technologiques, y compris celles qui ne sont pas retenues aujourd'hui comme prioritaires, leurs communautés et les débats qui les accompagnent pour réellement soutenir la transition.

Les chercheurs de SHS comme des autres sciences sont de plus en plus conscients de cette interpénétration nécessaire 
de problèmes scientifiques relevant ainsi concomitamment des compétences des uns et des autres. Il est d'ailleurs significatif que cette nouvelle vision globale de la science à laquelle les SHS adhèrent soit de plus en plus relayée par des projets institutionnels ou par des programmes.

Dans le cadre de cette vision globale, il serait inconcevable de prétendre traiter scientifiquement des enjeux auxquels la France et l'Europe sont confrontées sans les intégrer dans une perspective plus large, autour des aires culturelles non-européennes et non-atlantiques. Il s'agit d'une des forces majeures des SHS, notamment des humanités en France, et une des conditions de toute revendication d'universalisme. La connaissance de langues et civilisations, la compréhension du soi et de l'autre, des mentalités culturelles et des dynamiques religieuses sont des atouts considérables pour comprendre une mondialisation qui ne se limite pas aux flux financiers et aux échanges économiques et médiatiques, ni à une simple diffusion d'un unique modèle, mais suscite des stratégies de compétition entre ensembles dominants, qui jouent en particulier sur des déséquilibres financiers et les entretiennent.

Cette inscription dans l'espace, consubstantielle aux SHS, est indissociable de sa vision du monde dans le temps. L'étude des textes de l'Antiquité classique ou de l'époque médiévale fournit, par exemple, des arguments pour reconsidérer le modèle familial dominant depuis deux siècles. L'utilité sociale et politique de la recherche est, en l'occurrence, de souligner et de comprendre la variabilité des modèles familiaux au cours 
de l'histoire. Les sciences historiques françaises, en aspirant à affronter la mondialisation des échanges savants et universitaires, s'attachent à renforcer leur capacité à porter sur les autres sociétés un regard spécifique.

Les SHS portent de longue date une interrogation, souvent érudite, sur les assises de la civilisation occidentale, dont il faut désormais interroger les fondements implicites et les effets de domination. Elles sont aujourd'hui traversées par la question et l'exigence d'une désoccidentalisation des SHS.

Les SHS permettent ainsi une approche différenciée du développement de la connaissance et des cultures, et une dédramatisation du relativisme culturel. Un des progrès les plus marquants dans les recherches philologiques contemporaines est en effet la reconnaissance et l'approfondissement rigoureux des différences entre pratiques littéraires et théoriques engendrées par des univers linguistiques particuliers. C'est dans cette perspective que sont étudiés aussi bien les phénomènes de la traduction que les transferts conceptuels, constats d'incommensurabilité et d'intraductibilité, ou convergences surprenantes, qui permettent de bousculer quelques certitudes. La découverte des littératures non occidentales et le domaine émergent des études postcoloniales changent la donne, et permettront une réévaluation de nos hiérarchies culturelles.

Comme les autres sciences, les SHS sont sans frontières, qu'elles soient géographiques ou relevant des domaines de connaissance. Sur ce dernier aspect, c'est ce qu'illustre le 
développement de champs d'activité qui ont été reconnus au $\mathrm{XXI}^{\mathrm{e}}$ siècle comme faisant partie indissolublement de la culture et de la science : la performance et le spectacle vivant, la création artistique, l'architecture, le design, sont du domaine de la recherche, comme la musicologie et les arts plastiques, assurant la diversité et le décloisonnement des recherches en humanités. C'est d'ailleurs l'interdisciplinarité qui a permis une redynamisation de ces recherches, absentes par ailleurs de la plupart des universités : danse, voix, travail et reconstitution du son... Les nouvelles orientations technologiques et informatiques de la musicologie sont à la pointe du développement international de ces domaines. Les objets culturels en émergence, comme les performances practices et les performing arts en général, l'instrumentation appliquée, l'étude du geste musical et de l'improvisation, sont un haut lieu de la créativité des sciences, comme de l’innovation des humanités.

La recherche aujourd'hui doit prendre en compte la variété des formes d'innovation contemporaine dont font partie les arts vivants et les performances - tout ce qui permet le déplacement des hiérarchies et des certitudes de l'intellectualité traditionnelle, ébranlement qui a toujours été la source des avancées dans le les humanités. 


\section{Des SHS globales}

L'effet paradoxal de la mondialisation est que cette dernière pourrait donner à croire que sont devenus vains les efforts pour pénétrer les sociétés du monde à travers la connaissance de leurs langues et de leurs histoires longues.

La globalisation invite à penser autrement, à sortir du nationalisme méthodologique, à imaginer ce que peuvent être des sciences humaines et sociales non seulement à l'ère de la globalisation, mais elles-mêmes prises dans des logiques de globalisation. Faut-il rappeler ici que les sciences historiques françaises abordent depuis longtemps ces questions de circulations et de globalisation à l'échelle régionale ou mondiale (par exemple pour le monde romain ou l'Asie orientale) ? Penser global, c'est s’intéresser à des logiques planétaires, et articuler ce niveau d'analyse à d'autres niveaux régionaux, nationaux, locaux. C'est s'intéresser aux évolutions du droit contemporain, à la naissance d'un «droit global » qui, à certains égards, se substitue au droit international et parfois le complète, à la formation de contestations sociales, politiques ou culturelles de niveau mondial tout comme aux mobilisations locales, nationales ou régionales, telles que les mouvements altermondialistes. C'est considérer les phénomènes migratoires à la lumière, précisément, de la globalisation, et pas seulement dans l'optique de l'intégration normative à telle ou telle société d'accueil. C'est chercher à comprendre comment se développent des institutions supranationales, en matière de justice ou d'économie par exemple, tout comme en matière 
de régulation de la production énergétique ou d'action sur le climat. C'est aussi mettre au cœur de l'analyse les réseaux, qu'ils soient ou non fondamentalement tributaires d'Internet et des nouvelles technologies de communication.

Ce qu'on appelle changement global renvoie de fait à cette co-dépendance du monde humain et social ET du monde de la nature et de la terre. L'intégration des SHS permet l'analyse complexe et globale des conceptions et fondements normatifs sous-jacents à l'interprétation des risques liés aux changements globaux ; des modalités de la décision individuelle et collective en situation d'urgence sociétale : soutien aux politiques publiques, prise en compte des données, des « porteurs d'enjeux », de la société civile ; élaboration d'indicateurs nouveaux à portée sociale, sanitaire, environnementale. Elle permet l'élargissement des horizons de la recherche par la pratique renforcée de la comparaison dans l'espace et dans le temps, extrêmes compris, la prise en compte de l'innovation conceptuelle (de la perte de biodiversité à la justice spatiale, des situations de crise à la vulnérabilité, des capabilités individuelles et collectives aux inégalités de genre.)

Cette co-dépendance, qui a pour corollaire que des phénomènes locaux ont de plus en plus fréquemment des conséquences globales, ne peut être appréhendée sans prendre en compte les phénomènes d'émergence et d'auto-organisation propres aux systèmes technico-socio-économico-environnementaux. Les concepts et méthodes issus de l'étude des systèmes complexes sont en cela fondamentaux pour donner 
à la société une emprise sur des phénomènes qui tendent à acquérir leur propre autonomie.

L'aptitude des SHS, constitutive de leur vocation, à se situer à la fois dans le temps et dans l'espace, les prédisposent, à l'image de cet apport innovant des ouvres lointaines par le temps ou l'espace, à se confronter, en connaissance de cause, et en coordination avec les autres sciences, aux « défis » et enjeux des sociétés contemporaines et aux projections de leurs transformations dans l'avenir, qu'elles relèvent du physique, du naturel, du technologique ou du social, du politique et du culturel.

\section{La mobilisation de la science et de la société}

Les défis sociétaux expriment des urgences et difficultés globales; des questions auxquelles la recherche doit apporter aujourd'hui sinon des réponses, au moins des analyses ou des directions d'action : changement climatique, gestion des ressources naturelles, transition énergétique, protection des humains et de l'environnement, déferlante des big data et de leur traitement, risques sanitaires dans une économie mondialisée... sur lesquelles une mobilisation de l'ensemble des acteurs de la science est nécessaire.

Par sociétal, on doit entendre, nous l'avons vu, non pas un champ disciplinaire (les SHS), mais une dimension humaine, sociale, de la science. Le projet n'est pas d’intégrer les SHS 
aux autres secteurs scientifiques comme un complément immanent à chacun des défis. Au-delà des actions et structures existantes, il faut opérer un saut qualitatif et quantitatif dans l'articulation et l'intégration des compétences et contributions de diverses origines (par exemple, pour le défi énergie : physiciens, énergéticiens, chimistes, mais aussi sociologues, économistes, politistes, spécialistes du paysage, de l'environnement et de l'architecture... ; chercheurs, enseignants-chercheurs, industriels, petites et moyennes entreprises, associations, collectivités) en dépassant les hiérarchies implicites de la normativité académique.

$\mathrm{Au} \mathrm{XX}^{\mathrm{e}}$ siècle, la biologie a été révolutionnée par le perfectionnement des microscopes ; au XXI ${ }^{\mathrm{e}}$ siècle la convergence du big data et du calcul haute performance offre à toutes les sciences des outils nouveaux. Du fait de la médiation de pans entiers de l'activité sociale par les TIC, de nouveaux types de données (ex. : réseaux sociaux, traces GPS, données d'usage des services web, crowdsourcing) et des données d'une richesse inédite sont à disposition des chercheurs et de la société. Couplée avec des innovations technologiques et le développement de nouveaux formalismes pour l'étude des systèmes complexes (intégrant plusieurs niveaux d'organisation et de multiples échelles de temps et d'espace), cette transformation qualitative et quantitative de notre rapport au social est probablement l'une des clés de notre compréhension des phénomènes sociaux globaux. Ce couplage exige une synergie importante entre la recherche SHS et les recherches sur les systèmes complexes, ainsi 
qu'une mutualisation des compétences et des infrastructures, à l'échelle de la communauté scientifique. Une réflexion approfondie sur les bouleversements sociétaux qu'apportent inévitablement ces macroscopes s'impose donc à la science. 


\section{Conclusion}

Le régime de connaissance des sciences humaines et sociales a connu plusieurs transformations importantes. Le régime précédent était caractérisé principalement par une propension aux théories exclusivement critiques et à un alignement sur quelques grands paradigmes. La variété des sciences humaines était bien sûr présente mais elle s'accompagnait d'un fort cloisonnement des disciplines et sous-disciplines inscrites dans des traditions de savoirs et de domaines de savoirs utilisées comme instrument de légitimation du caractère infranchissable de leurs frontières. Elles n'excluaient pas le travail réflexif mais étaient principalement mobilisées contre le risque d'instrumentalisation ainsi qu'en témoigne la référence fréquente à la distinction de Max Weber entre la vocation du savant et celle du politique.

Le régime de connaissance qui s'impose désormais dans les formations et la recherche est marqué par une volonté d'interdisciplinarité fondée sur l'expérience acquise et des positions théoriques clarifiées. Le décloisonnement interne aux sciences sociales fait apparaitre tout le bénéfice intellectuel qui résulte 
de la capacité des formations et des démarches scientifiques à s'affranchir de piliers disciplinaires, qui résultent plutôt d'effets de traditions que d'impératifs méthodiques. L'ouverture de certaines sciences humaines et sociales au-delà du périmètre qui est le leur dans l'enseignement supérieur et la recherche est d'une tout autre nature. Elle requiert des processus d'appropriation et de croisement des raisonnements scientifiques propres aux études sur la matière et le vivant. C'est pourquoi, ce second type d'ouverture produit les plus grands déplacements, mais ne peut le faire que sur la base de protocoles localisés et maîtrisés. Ni l'une ni l'autre de ces démarches, interne ou externe, ne sauraient procéder d'une simple levée de barrières et sans autre finalité que l'adhésion idéologique au principe de l'ouverture.

Les conditions de l'innovation (culturelles, historiques, sociales, organisationnelles, économiques) sont ainsi toujours à mettre en avant dans les recherches transformatrices. Les dynamiques complexes des changements sociaux et économiques ne peuvent être séparées de l'étude des sociétés (pratiques sociales, partage des connaissances, dispositifs d'information et de participation, mobilisation de nouveaux acteurs). Il faut donc travailler sur les obstacles réels au changement: effets des inégalités liées à la nationalité, au genre, à la stratification sociale ou à l'origine sociale ; asymétries d'accès au savoir et à l’information. Les sciences humaines ne se réfèrent pas à la clôture de l'humain mais à la capacité de créer, cette capacité qui est au centre des dispositifs de production de richesses économiques. Cette capacité de créer implique le 
choix du détour, celui des rencontres improbables, celui de la surprise (invention, création) hors de toute assignation.

Une société au sein de laquelle naissent des innovations a dû préalablement mettre en place un système éducatif, scientifique, culturel et politique qui favorise les démarches innovantes: on ne peut rêver d'unîlot d'innovations techniques et scientifiques au milieu d'un océan de comportements traditionnels et routiniers. Il n'y a donc a priori aucune raison qu’une société refuse la nouveauté, sauf si elle ne correspond pas aux aspirations d'une part importante de la population. Le rôle des sociétés civiles, et non plus des seuls experts, dans la production de données ou de réflexions qui influent sur les orientations de la recherche doit être étudié et pris en compte. Les méthodes d'analyse des données, l'effort d'extraction de l'information pertinente, l'intervention des différents porteurs d'enjeux dans la décision... ont rendu obsolètes les approches de la décision dite «evidence-based », fondées sur des modèles déductifs individualistes qui ont, en leur temps, constitué un progrès par rapport aux techniques intuitives. Dans de nombreuses organisations sociales, c'est bien la multitude qui est productive, porteuse d'innovations et de dynamiques collectives et qui interroge le chercheur qui ne peut s'enfermer dans sa tour d'ivoire. Affronter les défis sociétaux en défendant des collaborations et interactions inédites entre les scientifiques, les entreprises, les associations, les collectivités, toutes les parties prenantes, c'est promouvoir l'évolution dynamique d'une science qui sera d'autant plus ouverte qu'elle sera respectée dans ses fondements, ses principes et ses pratiques. 
Si l'importance de la crise actuelle, sociale, économique et institutionnelle engage à ouvrir les perspectives en évitant le réductionnisme a-historique, le normativisme autoritaire et toute approche nationalo-centrée ou européano-centrée, l'abondance de la production scientifique et l'organisation des systèmes éducatifs œuvrent en faveur de spécialisations très fines. Face à l'émiettement des savoirs, l'éducation a un rôle plus structurant que jamais. Il convient ainsi d'encourager toutes les mesures qui visent à doter les étudiants d'une culture indispensable au développement de l'esprit critique. La recherche sur l'éducation doit être relancée. Elle devra se pencher sur l'analyse des failles du système éducatif, sur l'exploration de nouvelles ressources pour l'éducation en lien avec le renforcement des inégalités. Des doubles cursus, et cursus interdisciplinaires en SHS et autres sciences, devraient être créés en liaison avec les grands défis de la société.

Comme toutes les autres sciences, les SHS sont portées par la dialectique de la capitalisation de masses énormes d'acquis et du renouvellement de leurs paradigmes et de leurs méthodes. Cette dialectique est d'autant plus délicate aujourd'hui qu'elle oblige à éviter en permanence le poids de la tradition (ressassement des cadres théoriques éprouvés mais qui ne sont plus en prise avec les réalités nouvelles) et les écrits de circonstance (essais, textes d'humeur, qui peuvent être des objets pour la science mais pas des productions scientifiques). Les nouvelles pratiques d'évaluation du travail scientifique peuvent décourager ce travail en profondeur et incitent à exploiter des « filons disciplinaires » à l'intérieur desquels 
le travail de distinction entre texte d'opinion et écrit scientifique est faible. Le mouvement de repli disciplinaire se trouve ainsi soutenu par ces pratiques comme par celles qui prônent le cloisonnement géographique. Il revient à la communauté scientifique de retrouver ses prérogatives en la matière et de proposer, dans un dialogue renouvelé avec leurs institutions, des approches plus pertinentes au regard de l'exercice scientifique.

L'efficacité de l'ensemble du système repose dès lors sur la composition harmonieuse entre trois types de performances, dont il faut veiller à n'investir aucune d'elle d'une légitimité superlative : les performances patrimoniales qui sont garantes de la qualité et de l'efficacité du travail raisonné de conservation, de classement, de reconstitution, de traduction de l'héritage culturel de l'humanité ; les performances incrémentales qui apportent la démonstration du caractère cumulatif des résultats qu'elles produisent, en sachant distinguer les simples redites, d'un côté, et les effets de seuil produits par l'approfondissement des connaissances, d'un autre côté, et en s'appuyant sur cette distinction pour modifier les approches et les méthodes ; les performances d'innovation qui encouragent les expérimentations et les questionnements inattendus et apprennent à gérer positivement les risques qu'entraîne l'exploration de l'inconnu.

Au-delà des sciences humaines et sociales, les évolutions des sociétés invitent à renouer avec la conception formulée par les premiers théoriciens des sciences de la société qui ont 
dessiné une figure de «l'ingénieur » largement empreinte d'une formation ouverte aux humanités. Le temps est venu de mettre un terme à l'hyperspécialisation du chercheur et de l'enseignant. D’autres voies sont à promouvoir pour faire des hommes et des femmes de science qui évitent les grands écueils de l’intolérance, du dogmatisme et du scientisme. 




\section{5 principes d'action pour la recherche en Europe}

1. Remettre au centre des préoccupations des politiques scientifiques la question du lien entre la Formation et la Recherche en promouvant, pour l'ensemble des secteurs, des formations ouvertes aux SHS.

2. Penser le soutien à la recherche sur projets en tenant compte des besoins d'une science inscrite dans la durée.

3. Renforcer les dispositifs de circulation des compétences entre les différents domaines scientifiques sur l'ensemble du territoire européen.

4. Soutenir des infrastructures de recherche en SHS à la hauteur des enjeux du numérique.

5. Accompagner le formidable potentiel de jeunes chercheurs en leur offrant de nouvelles opportunités professionnelles. 



\section{Comité d'orientation}

Le Comité d'orientation de l'Alliance ATHENA rassemble plus de 50 scientifiques œuvrant à la structuration et au pilotage de la recherche SHS en France. Il s'est réuni par deux fois au cours de l'année 2013/2014 dans le cadre du séminaire de Paris et du séminaire de Caen. Cet ouvrage n'aurait pu voir le jour sans les réflexions, interrogations et discussions des participants de ces séminaires :

Arnaud Pascal Professeur - Université de Lyon 2 Anheim Etienne Maître de conférences - Université de Versailles Arena Richard Professeur - Université de Nice Audiffren Michel Professeur - Université de Poitiers Baussant Michèle Chargée de recherche - CNRS, MAE Nanterre, Directrice adjointe scientifique CNRS-InSHS

Béjean Sophie Présidente du CNOUS et de Campus France Benamouzig Daniel Chargé de recherche - CNRS Bonnafous-Boucher Maria Conseiller adjoint de coopération et d'action culturelle, HDR - Université de Paris Dauphine

Bouin Olivier Directeur du Réseau français des Instituts d'Etudes Avancées

Bourdelais Patrice Directeur de l'INSHS du CNRS Buléon Pascal Directeur de recherche - CNRS 
Bungener Martine Directrice adjointe du CERMES 3

Casella Philippe Directeur du développement de la recherche - EHESS

Cases Chantal Directrice de l'INED

Caverni Jean-Paul Vice-président délégué de l'Idex d'AixMarseille Université

Conrad Christoph Professeur - Université de Genève

Courtet Catherine Coordinatrice scientifique, département sciences humaines et sociales - ANR

Di Meo Guy Professeur - Université de Bordeaux 3

Dubucs Jacques Directeur de recherche - CNRS, Directeur scientifique - DGRI

Dumasy Lise Présidente de l’Université de Grenoble 3

Filliatreau Ghislaine Directrice de recherche - INSERM

Fremont Armand Géographe

Gaffard Jean-Luc Professeur - SKEMA Business School

Garden Maurice Historien, Professeur honoraire des Universités Gombert Jean-Emile Président de l'Alliance Athena, Président de l’Université Rennes 2

Krasteva Anna Professeure - Nouvelle université bulgare Hatchuel Armand Professeur - Ecole des mines de Paris Hautcoeur Pierre-Cyril Président de l'Ecole des Hautes Etudes en Sciences Sociales

Jouve Bertrand Professeur - Université Lyon 2, Directeur adjoint scientifique CNRS-InSHS

Kirman Alan Directeur d'étude - EHESS

LaugierSandra Professeure-UniversitéParis I Panthéon Sorbonne, membre de l'IUF, Directrice adjointe scientifique CNRS-InSHS Lemaître Denis Professeur - ENSTA Bretagne 
Lesourne Jacques Professeur émérite au CNAM

Livet Pierre Professeur émérite - Aix-Marseille 1

Lussault Michel Professeur - Ecole Normale Supérieure de Lyon

Maillard Christine Professeure-Université de Strasbourg

Marin Brigitte Professeure - Université Aix-Marseille I

Marty Pascal Professeur - Université de la Rochelle, Directeur adjoint scientifique CNRS-InSHS

Marx William Professeur - Université Paris Ouest Nanterre La Défense

Mespoulet Martine Professeure - Université de Nantes

Musselin Christine Directrice du Centre de Sociologie des Organisations

Petit Antoine Directeur général adjoint de l'Inria

Peyraube Alain Directeur de recherche, EHESS

Ranc Emmanuel Directeur du Réseau National des Maisons des

Sciences de l'Homme

Randet Denis Délégué général de l'ANRT

Rouillard Pierre Directeur de recherche - CNRS / MAE

Ruggiu François-Joseph Professeur - Université Paris Sorbonne,

Directeur adjoint scientifique CNRS-InSHS

Senut Brigitte Professeure - Museum national d'histoire naturelle

Pierre Sineux Président de l'Université de Caen

Vandelac Louise Professeure - Université du Québec

Vendrix Philippe Directeur de recherche - CNRS, Président du Réseau Nationale des Maisons des Sciences de l'Homme

Wallerstein Immanuel Sociologue

Wieviorka Michel sociologue, Administrateur de la Fondation

Maison des Sciences de l'Homme

Wolikow Serge Professeur - Université de Bourgogne 



\section{Remerciements}

Cet ouvrage a bénéficié des précieux conseils, critiques et contributions de Maria Bonnafous-Boucher, Michèle Baussant, Pascal Buléon, Olivier Bouin, Maurice Garden, François Héran (Directeur du département des sciences humaines et sociales de l'ANR), Sandra Laugier, MarieClaire Lavabre (Directrice de recherche au CNRS), Pierre Moeglin (Professeur, membre de l'IUF), Pierre Rouillard et Jean-Frédéric Schaub (Directeur d'étude à l'EHESS),

Nos remerciements vont également à la Bulac qui a hébergé le séminaire de Paris en 2013 et à la Maison des Sciences de l'Homme de Caen, son directeur Pascal Buléon et l'IMEC, qui ont permis la tenue du séminaire de Caen en février 2014.

Enfin, nous remercions très chaleureusement Marianne van de Logt qui a grâcieusement mis à disposition ses oeuvres pour l'illustration de cet ouvrage. 
Achevé d'imprimer en octobre 2014 sur les presses de l'Imprimerie de l'Ouest Parisien 92100 Boulogne-Billancourt

Dépôt légal : octobre 2014

Imprimé en France 



\title{
Des sciences dans la Science
}

\author{
Ouvrage dirigé par \\ Jacques Commaille \& Françoise Thibault
}

Dans un contexte européen où se redéfinissent les grandes orientations de la recherche, une réflexion s’impose sur les principes généraux au fondement des sciences humaines et sociales et sur leur place dans la science et dans la société.

La nécessité d’un dialogue fécond entre les sciences pour faire face aux grands enjeux du monde contemporain, invite à renouer avec la figure de «l'ingénieur » largement empreinte d'une formation ouverte aux humanités.

Au-delà des considérations propres au monde scientifique, les SHS reposent la question du sens même de nos sociétés et de leurs évolutions.

Fruit d'une mobilisation exceptionnelle de chercheurs et enseignants, cet ouvrage remet les SHS à la place qui leur revient et encourage la promotion d'hommes et de femmes de science qui évitent les grands écueils du technicisme, du dogmatisme et du scientisme.

Jacques Commaille - Professeur émérite à l'Ecole Normale Supérieure de Cachan et Président du Comité d'orientation de l'Alliance ATHENA

Françoise Thibault - Directrice de programme à la Fondation Maison des Sciences de l'Homme et Déléguée générale de l’Alliance ATHENA.

\section{Collection ATHENA}

